\title{
XLVII.
}

\section{Ueber den Aethylamyläther und über Aetherification.}

\author{
Von \\ C. Friedel und J. M. Crafts. \\ (Compt. rend. t. LVH, p. 986).
}

In vorstehender Abhandlung führten wir an, dass sich bei Einwirkung von Jodäthyl auf Amylalkohol auch der zusammengesetzte Aethylamyläther bildet. Um denselben $\mathrm{zu}$ isoliren, sammelten wir die zwischen 100 und $120^{\circ}$ siedenden Producte, welche bei dieser Einwirkung entstehen; sie enthielten viel Jodüre. Wir erhitzten sie so lange mit Natrium in einem mit aufgesetztem Kühlrohr versehenen Ballon bis dieses keine Wirkung mehr auf die Flüssigkeit zeigte und destillirten dieselbe dann. Nach zwei oder drei Operationen erhielten wir eine gewisse Menge einer klaren, angenehm ätherisch riechenden Flüssigkeit, welche zwischen 110 und $113^{\circ}$ siedete und bei der Analyse Zahlen gab, welche der Formel des Aethylanyloxyds $\left.\begin{array}{c}\epsilon_{2} \mathrm{H}_{5} \\ \boldsymbol{C}_{5} \mathrm{H}_{11}\end{array}\right\} \boldsymbol{\theta}$ entsprechen. Dasselbe siedet nach Williamson bei $112^{\circ}$.

Als wir denselben Versuch mit gewöhnlichem Alkohol und Jodamyl machten erhielten wir gleichfalls einen gemischten Aether. Das Product enthielt etwas zu viel Kohlenstoff, was leicht erklärlich ist, denn das Gemisch, aus welchem man in diesem Falle den gemischten Aether auszieht enthält eine viel grössere Menge Jodamyl und dieses letztere giebt durch seine Wirkung auf Natrium Amyl, das, wenn auch nur in sehr geringer Menge vorhanden, den Kohlenstoffgehalt der analysirten Substanz beträchtlich erhöht.

Die Bildung des Aethylamyläthers unter diesen Umständen ist übrigens leicht einzusehen; Jodäthyl und Amylalkohol reagiren beide aufeinander, wie in Williamson's 
Versuch das Jodäthyl auf Aethernatron einwirkt; es wird Jodwasserstoffsäure frei und diese giebt mit dem überschüssigen Amylalkohol, Jodamyl und Wasser:

$$
\left.\left.\underset{\mathrm{H}}{\mathrm{\epsilon}_{5} \mathrm{H}_{11}}\right\} \boldsymbol{\theta}+\mathrm{\epsilon}_{2} \mathrm{H}_{5} \mathrm{~J}=\underset{\epsilon_{2} \mathrm{H}_{5}}{\stackrel{\mathrm{C}_{5} \mathrm{H}_{11}}{3}}\right\} \boldsymbol{\theta}+\mathrm{HJ}
$$

Das Wasser kann dann im weitern Verlaufe die Jodüre des Aethyls und Amyls zersetzen nnter Bildung der entsprechenden Alkohole.

Die Bildung der Jodwasserstoffsäure und des Wassers ist nicht hypothetisch. Wir haben schon in vorstehender Abhandlung die Abscheidung einer gewissen Menge Wasser auf der Oberfläche der gemischten Flüssigkeiten nach der Reaction angeführt und haben uns überzengt, dass dieses Wasser beträchtliche Mengen freier Jodwasserstoffsäure enthält.

Man weiss, dass das Chlor-, 'Jod- und Bromäthyl die Eigenschaft haben, eine fast unbegrenzte Menge Alkohol in Aether umzuwandeln, was von Vielen einer Contactwirkung zugeschrieben wird, während Andere, wie z. B. Reynoso diese Zersetzung einer successiven Zersetzung und Wiederneubildung von Jodüthyl zuschreiben. Die Bildung des gemischten Aethylamyläthers spricht entschieden für letztere Ansicht.

Aber nicht nur auf die organischen Chlorüre, Jodüre und Bromüre lässt sich diese Erklärung anwenden, sie passt auch vollkommen auf die metallischen Chlorüre, Jodüre, Bromüre und Sulfate.

Pasteur hat in seinen schönen Untersuchungen über die Gährung gezeigt, dass die Contactwirkung des Ferments keine andere als eine physiologische Wirkung ist, die nacheinander auf im Verhältniss zum Ferment in sehr grosser Menge vorhandene Substanz ausgeübt wird. In unserem Falle scheint es sich ganz ähnlich zu verhalten. Eine sehr geringe Menge von Jodäthyl z. B. kann durch ihre fortwährende Zersetzung und Wiederbildung eine grosse Menge Alkohol in Aether überführen und das Jodür findet sich zu Ende der Operation in fast unveränderter 
Menge wieder. Die Contactwirkung ist daher in diesem Falle eine rein chemische nach und nach stattindende Wirkung.

\section{XLVIII. \\ Ueber Darstellung von Bromacetyl, Wirkung von Brom auf dasselbe und über Tribrom- essigsäure.}

Das Verfahren, dessen man sich gewöhnlich bei Darstellung des Bromacetyls bedient besteht darin, dass man Phosphorsuperbromid auf Eisessig wirken lässt, nach der Gleichung:

$$
\mathrm{C}_{8} \mathrm{H}_{4} \mathrm{O}_{4}+\mathrm{PBr}_{5}=\mathrm{PO}_{2} \mathrm{Br}_{3}+\mathrm{HBr}+\mathrm{C}_{6} \mathrm{H}_{3} \mathrm{O}_{2} \mathrm{Br} \text {. }
$$

Von den 5 A eq. Brom tritt also nur eins in das Präparat, ausserdem ist die Darstellung des Phosphorbromid sehr mühsam und die Trennung des Bromacetyls vom Phosphoroxybromür nicht immer vollständig.

H. Gal hat bei Versuchen in Cahours' Laboratorium an der École polytechnique folgendes einfacheres Verfahren gefunden, welches sehr gute Resultate giebt (Compt. rend. t. LVI, p. 1257). Man bringt in eine Retorte 3 Aeq. Eisessig und 2 Aeq. rothen Phosphor und giesst dureh eine ausgezogene Röhre 6 Aeq. Brom darauf. Die Reaction ist sehr energisch und die Entfärbung eines jeden Tropfen Brom angeriblicklich. Es entwickelt sich Bromwasserstoff und gleichzeitig entsteht phosphorige Säure und Bromacetyl deren Trennung durch Destillation sehr leicht ist. Folgende Gleichung drückt den Vorgang aus:

$$
3 . \mathrm{C}_{4} \mathrm{H}_{4} \mathrm{O}_{4}+2 . \mathrm{PBr}_{3}=3 . \mathrm{C}_{4} \mathrm{H}_{3} \mathrm{O}_{2} \mathrm{Br}+3 . \mathrm{HBr}+2 . \mathrm{PO}_{3} \text {. }
$$

Die Hälfte des angewendeten Broms dient zur Darstellung des Bromacetyls. Mit 240 Grm. Brom, 90 Grm. Essigsäure und 33 Grm. Phosphor orhielt der Verf. 140 Grm. bei $81^{\circ}$ siedendes Bromacetyl. 